\title{
Statistical estimates of the transcription factor binding sites cluster in Arabidopsis and distant plant species genomes
}

Orlov Y.L. ${ }^{1,2 *}$, Dergilev A.I. ${ }^{1}$, Dobrovolskaya O.B. ${ }^{2,3}$

${ }^{1}$ Novosibirsk State University, Novosibirsk, Russia

2 Peoples' Friendship University of Russia (RUDN University), Moscow, Russia

${ }^{3}$ Institute of Cytology and Genetics, SB RAS, Novosibirsk, Russia

*email: orlov@bionet.nsc.ru

The development of high-performance sequencing technologies allows to study the binding sites of protein transcription factors in genome scale. The clusters of transcription factor binding sites determine regulatory gene networks and evolutionary patterns. The growth of data volume on the experimentally determined binding sites raises qualitatively new problems for the analysis of gene expression, determining target genes for transcription factors. However, such data were not investigated in plant genomes in detail comparing to mammalian genomes. Plant genomes remain an insufficiently studied object, although they have complex molecular regulatory mechanisms of gene expression and response to the environmental stresses. It is important to develop new software tools for the analysis of the transcription factor binding sites location, their clustering in a model genome, visualization, and statistical estimates for such clusters. The existence of non-random clusters of the binding sites for 3 and more different factors identified by ChIP-seq was shown previously. Such clusters of sites could be used for gene promoter and enhancer prediction. This work presents a new application for the analysis of transcription factor binding sites in several evolutionarily distant model plant organisms. This work presents the applications of computer scripts to analyze ChIP-seq data, description of clusters and visualization in the heatmaps format. We used ChIP-seq profile peaks to study the transcription factor binding in three plants, including Arabidopsis thaliana, Physcomitrella patens, and Chlamydomonas reinhardtii. We discussed statistical estimates of the binding sites clusters found. The nonrandom clusters of binding sites in the investigated plant genomes are shown; and binding clusters in Arabidopsis were considered in more details including gene ontology and functional annotation. 